\title{
The Three-Level Approach: A Framework for Ensuring Medicines Quality in Limited-Resource Countries
}

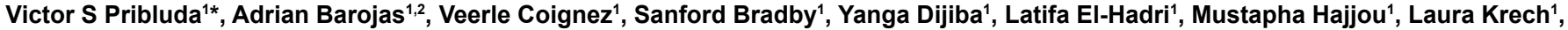 \\ Souly Phanouvong ${ }^{1}$, Karim Smine ${ }^{1}$, Kennedy Chibwe ${ }^{1}$, Patrick H Lukulay ${ }^{1}$ and Lawrence Evans III ${ }^{1}$
}

${ }^{1}$ Promoting the Quality of Medicines Program, Global Health Impact Programs, United States Pharmacopeial Convention, Rockville, MD, USA

${ }^{2}$ Currently at the Pan American Health Organization, Washington, D.C., USA

\begin{abstract}
Regulators from countries at all levels of income struggle to protect the public from the dangers of poor-quality (counterfeit and substandard) medicines. In particular countries with limited resources are at higher risk because of weak regulations, insufficient personnel, or laboratories with poor infrastructure and a lack of equipment required to perform quality control analysis. A systematic approach is needed to address these gaps.

A stepwise process was used to design medicine quality monitoring programs in numerous countries in Africa, South America, and Southeast Asia. The experience gained in these countries resulted in the development of the Three-level Approach for performing quality control of medicines throughout the supply chain.

The approach consists of three successive, complementary, and increasingly complex levels of analysis: The first level includes visual and physical inspection to assess package and insert conditions and information as well as the physical characteristics of the actual medicines. The second level consists of rapid analytical tests that assess a limited number of quality attributes and can easily be performed in the field by trained personnel. The third level involves quality control testing according to the product's registration specifications and is performed in an appropriate laboratory setting by experienced and trained analysts.

The Three-level Approach offers regulators in limited resource countries, a cost-effective high-throughput methodology for quality monitoring that produces valid and trustworthy results. The approach strengthens medicines quality assurance systems by allowing better regulation of the pharmaceutical market, which ultimately reduces the prevalence of poor-quality medicines.
\end{abstract}

Keywords: Quality of medicines; Quality control; Analytical tests; Counterfeit; Pharmaceutical market

\section{Introduction}

The availability and use of medicines for the treatment of diseases are basic components of any health care system, and to treat illnesses effectively, medicines must provide the desired therapeutic effect and have an acceptable safety profile, both of which depend on the medicines' being of the appropriate quality.

Poor-quality medicines are those that do not conform to the standards established, usually during registration. The World Health Organization (WHO) describes poor-quality medicines as substandard or spurious, falsely labeled, falsified, or counterfeit (SFFC) [1]. Poorquality medicines pose a serious threat to consumers, and result in economic losses that in resource-limited countries waste financial resources that could be used for other priority areas of health care $[2,3]$. Moreover, as is the case with infectious diseases such as malaria, HIV/AIDS, and tuberculosis, poor-quality medicines may contribute to the development of antimicrobial resistance, impairing currently available treatments $[2,4]$. Ineffective treatment caused by poor-quality medicines may also cause a loss of confidence in medicines and in the national health care system [5].

The likelihood of access to poor-quality medicines is higher in countries with limited resources [6-12], due in part to weak legislation and regulatory systems, ineffective national Medicines Regulatory Authorities (MRA), high cost of medicines, or insufficient access to formally regulated dispensaries in rural or decentralized areas $[2,6,13,14]$.

A country's MRA must adequately control and regulate its entire pharmaceutical market, and to carry out its responsibilities effectively, it must establish a Quality Assurance (QA) system that ensures the quality of medicines throughout the pharmaceutical supply chain- from manufacturing to consumer use. Within the QA system the MRA must have access to a laboratory, typically the country's Official Medicines Control Laboratory (OMCL), which can properly perform Quality Control (QC) analysis to verify that medicines comply with appropriate quality standards.

Medicines' QC is important both, during registration for marketing authorization and after the authorization has been granted. After marketing authorization is granted, QC could be performed before distribution, as is the case for national health programs (e.g. Malaria, HIV/AIDS, Tuberculosis) B and/or through Medicines Quality Monitoring (MQM) activities when medicines are already in the market. The latter is particularly important because in many countries, during registration, the MRA perform only a documentary review of quality control data provided by the manufacturer. A WHO assessment [15] found that 14 of 26 MRAs surveyed lacked an MQM program, although organizations such as Medicines for Malaria Venture have publicly advocated for them [16]. The absence of MQM programs leaves these countries vulnerable to poor-quality medicines resulting

*Corresponding author: Victor S Pribluda, Promoting the Quality of Medicines Program, Global Health Impact Programs, United States Pharmacopeial Convention, 12601 Twinbrook Parkway, Rockville, MD, 20852-1790, USA, Tel: 1-301-816-8258; E-mail: vsp@usp.org

Received February 13, 2014; Accepted February 23, 2014; Published March 04 2014

Citation: Pribluda VS, Barojas A, Coignez V, Bradby S, Dijiba Y, et al. (2014) The Three-Level Approach: A Framework for Ensuring Medicines Quality in Limited-Resource Countries. Pharmaceut Reg Affairs 3: 117. doi:10.4172/21677689.1000117

Copyright: @ 2014 Pribluda VS, et al. This is an open-access article distributed under the terms of the Creative Commons Attribution License, which permits unrestricted use, distribution, and reproduction in any medium, provided the original author and source are credited. 
from poor manufacturing, deficient control during registration, improper trading practices and/or introduction of SFFC. Of the 12 medicine regulatory authorities with some form of MQM program included in the aforementioned WHO study [15], less than $20 \%$ used a systematic approach for costly QC testing. Governments sometime pay for MQM activities by means of budget allocations and MRA's service fees; however, fees charged by governments in numerous countries are frequently insufficient to support MRA activities [17].

Because of the limited availability of human and financial resources, it may be difficult in many low-and low-middle income countries to ensure the quality of medicines throughout the supply chain by relying exclusively on the OMCL for QC activities. Therefore, it is in the best interest of those countries to explore novel cost-effective mechanisms for the MRA to maximize the use of their scarce resources. The Promoting the Quality of Medicines (PQM) program [18], working through a collaborative agreement between the United States Pharmacopeial Convention (USP) and the US Agency for International Development (USAID), developed the Three-level Approach described in this article to address financial and human limitations frequently encountered to perform QC of medicines. The approach has been successfully applied in many countries in Africa, South America, and Southeast Asia. The success achieved in those countries provided the necessary evidence to propose its implementation beyond the countries where PQM operates. Though its implementation would be most beneficial where human and financial resources are limited, it may be applied for quality control of medicines throughout the supply chain in any country.

\section{Components of the Three-level Approach}

The stepwise process utilized by PQM for MQM builds on the basic QA model that relies on an MRA and an OMCL at its center. Without introducing novel analytical methodologies, the approach extends medicines QC beyond an established laboratory by the systematic and successive initial use of two additional levels of assessment-visual/ physical inspection and screening testing.

A brief description of the three levels is provided in the following sections and a summary of each level is included in Table 1 .

\section{Level 1 (L1) analyses}

Visual and physical inspection, are a quick and easy way to detect poor-quality medicines. Visual inspection focuses on labeling, packaging integrity, and package information, and physical inspection centers on its appearance. Both ensure that critical quality attributes (name of product, list of active ingredients, dosage form, number of units, date of expiry, storage and handling conditions, warnings, and directions for use, etc.) comply with the appropriate quality standards, regulatory requirements, and registration specifications. Visual and physical inspection, which is traditionally assessed as the first step in the analysis performed at the lab, in the Three-level Approach stands by itself as the sole component of L1. L1 analyses can be performed at every stage of the medicine supply chain, even by the patient, before use.

Visual inspection may identify medicines that have been tampered with, altered, or damaged during any stage of a medicine's life cycle. It authenticates packaging by checking for defective packaging and suspicious or missing information about the medicine's manufacture, distribution, dosage form, strength, or expiration date.

Physical inspection focuses on the physical characteristics of a medicine and varies according to dosage form. For instance, tablet and capsule dosage forms may be evaluated in terms of their shape, size, color, and texture, whereas liquids or injectables may be inspected for color or presence of particulate matter. In either case, physical inspection identifies shortcomings that may be the result of inadequate manufacture, damaged packaging, or deficient storage conditions.

\section{Level 2 (L2) analyses}

Various screening tests could be used to perform L2 analyses. Several methodologies are currently available for this purpose, such as Raman spectroscopy, infrared spectroscopy, Thin-Layer Chromatography (TLC) and, for solid dosage forms, disintegration. Taking into account price, availability of required supplies and extent of information that can be gathered, PQM currently recommends for this level the use of TLC and disintegration.

1. TLC assesses three critical quality attributes: identity, content, and impurity. Identity tests ensure that a medicine contains the Active Pharmaceutical Ingredient (API) stated on the product's label. Content is assessed visually by comparison of the intensity of the spots of the API in the sample and in a reference standard, allowing a semi-quantitative estimation within a limited range (e.g., $\pm 20 \%$ ) of the amount declared in the product labeling; however, use of portable densitometers could provide a more accurate value. Impurity tests can determine if the medicine contains spurious substances appearing as spots not present in the standards, which could be toxic, decrease the effectiveness and/or signal degradation of the API. Products that do not comply with identity or content requirements commonly are the result of poor manufacturing or counterfeiting, the presence of impurities may also result from poor manufacturing including the use of poor-quality raw materials, or product degradation following exposure to environmental conditions outside of manufacturer's specifications. A brief illustrative description of this test and how it may be applied in the field may be found at GHFPMinilab Manual [19].

\begin{tabular}{|c|c|c|c|c|}
\hline Level & Type of Analysis & Type of Test & Purpose & Site Personnel Responsible \\
\hline 1 & $\begin{array}{l}\text { Visual \& Physical } \\
\text { Inspection }\end{array}$ & $\begin{array}{l}\text { - } \quad \text { Labeling and packaging properties } \\
\text { - } \quad \text { Appearance, conditions and } \\
\text { physical characteristics of } \\
\text { medicine }\end{array}$ & $\begin{array}{l}\text { Identify expired medicines and/or medicines with } \\
\text { insufficient, erroneous and/or fraudulent information; } \\
\text { damage to packaging; damage and/or alterations to } \\
\text { the condition of the medicines }\end{array}$ & $\begin{array}{l}\text { Management staff at every } \\
\text { stage of the supply chain, from } \\
\text { procurement to use, including } \\
\text { patients }\end{array}$ \\
\hline 2 & $\begin{array}{l}\text { Simple, Rapid and Cost } \\
\text { Effective Screening } \\
\text { Tests }\end{array}$ & $\begin{array}{ll}- & \text { Thin layer chromatography }(T L C) \\
- & \text { Disintegration } \\
- & \text { Colorimetric reactions }{ }^{1} \\
- & \text { Spectroscopy based technologies }\end{array}$ & $\begin{array}{l}\text { Identify medicines deficient in at least four critical } \\
\text { quality attributes }{ }^{2} \text { (identity, content, impurities, and } \\
\text { disintegration for solid dosage form) }\end{array}$ & $\begin{array}{ll}- & \text { Personnel trained in } \\
& \text { Screening Tests } \\
- & \text { OMCL personnel }\end{array}$ \\
\hline 3 & $\begin{array}{l}\text { Pharmacopeial or } \\
\text { Manufacturer's Validated } \\
\text { Tests }\end{array}$ & According to registration specifications & $\begin{array}{l}\text { Assessment of all critical quality attributes of the } \\
\text { medicine }\end{array}$ & OMCL personnel \\
\hline
\end{tabular}

${ }^{1}$ The use of colorimetric methods is not recommended when a TLC method is available for the same medicine.

Table 1: Three-level Approach. 
2. Colorimetric methods may be employed for L2 testing. However, they serve only for identity tests, because content may not be assessed without additional equipment that is not usually available for field use.

3. Disintegration tests determine the ability of solid oral dosage forms (e.g. tablets and capsules) to break apart in a particular medium, at a specific temperature, and within an established time period. A failed disintegration test predicts that the medicine will not be efficacious in its intended use because will deliver less than the required amount of API. Products that do not comply with disintegration tests are commonly the result of poor manufacturing procedures or exposure to environmental conditions outside the manufacturer's specifications. Though disintegration is not a proxy for dissolution, it is a critical step to attain complete dissolution.

4. Spectroscopic technologies employed in L2 analyses are used primarily to assess identity using distinct chemical properties of a medicine via interactions with light, resulting in a unique signal. In recent years, portable devices using this technology have become available but are much more expensive than other techniques and may require a spectral library for comparison. Further, readings from these devices may be affected by formulation differences, and problems have been found in their ability to detect substandard medicines [20].

\section{Level 3 (L3) analyses}

L3 analyses uses the most stringent tests and are the only ones that can assess compliance with the quality attributes and specifications established during registration. These analyses are capable of evaluating content and impurities with more accuracy than those described above for $\mathrm{L} 2$ testing. It would be beyond the scope of this article to detail the diversity of tests included in Level 3, because they vary significantly not only with the dosage form but also, within a dosage form, with the particular product. MRAs in most countries recognize international pharmacopeia methodologies (British Pharmacopeia, European Pharmacopeia, International Pharmacopeia, Japanese Pharmacopeia, and United States Pharmacopeia) and access to those is readily available. The most common L3 analyses that apply to practically all dosage forms are tests for identification, strength/content (assay), and impurities (organic, inorganic, and/or residual solvents). However, critical quality attributes examined by L3 analyses also depend on the dosage form being evaluated. In the case of solid oral dosage forms, requirements may include dissolution, uniformity of dosage units, and water content; while for injectables usual requirements are microbiological tests (eg, sterility, pyrogenic activity) and particulate matter.

In most countries, the methods used in L3 analyses (manufacturervalidated or pharmacopeial methodology) are the legally accepted standards for quality required by national regulations.

\section{Discussion}

\section{Advantages and limitations of the staged incorporation of L1 and $\mathrm{L} 2$ analyses for QC}

The introduction of L1 (Visual and physical inspection) and L2 (Rapid screening tests, mainly disintegration and TLC in the context of this article) as intermediate self-standing QC stages is the main novelty of the Three-level approach. The discussion, based on PQM field experience, will be mostly focused on L2 testing as opposed to those used in L3 (registration specific tests, which as explained above are either manufacturer's validates methods or readily available pharmacopeial methodologies).

\section{Advantages:}

1. Access in the field: In decentralized areas where prompt access to the OMCL is not possible, the systematic in situ implementation of L1 or L1 and L2 testing offers a reasonable approach to address the quality of medicines that otherwise could remain unattended.

2. Capability to identify a large proportion of poor-quality medicines: Identity, content and impurities, three of the four critical quality attributes assessed through L2, are the most frequent reasons why medicines fail quality standards.

3. Reliability of results: When L2 tests are properly performed, the results are highly reproducible and valid within the stated limits [21].

4. Timely implementation of corrective actions: Regulations in most countries require L3 testing before the MRA can withdraw and destroy poor quality medicines. However, getting results from L3 tests may be a lengthy process, during which the suspicious products continue to be consumed by the uninformed public. Staged L1 and L2 testing as part of MQM would allow the MRA to rapidly identify potential risky products, which could be withdrawn immediately from circulation and quarantined until the definitive confirmatory L3 tests are available.

5. Large analytical throughput: Our experience has shown that with L2 methods it is feasible to analyze in situ a large number of samples in a very short period of time, many more than with L3 analyses. Screening samples in the field eventually reduces the number of samples that must be tested at OMCLs, which allows a more efficient use of OMCL resources and, ultimately, broader sampling of the pharmaceutical market.

6. Minimal infrastructure requirements: The equipment and tools necessary to perform L2 analyses are minimal, readily accessible, and of low cost. In addition, a pre-assembled portable kit (Global Pharma Health Fund (GPHF) Minilab") [22] that includes detailed methodologies, reagents and supplies is available. This means that L2 can be applied in a broad range of locations, expanding the geographical reach of the MRA.

7. Minimal need of specialized human resources: L2 testing is simple and straightforward and personnel with varying backgrounds (high school graduates, university students, less-experienced chemists, and others) can be trained to perform these analyses effectively.

8. Low acquisition and maintenance costs: The cost of procuring and maintaining the equipment, tools, and supplies necessary to perform L2 tests is low compared to the cost of equipment and reagents required for pharmacopeial tests at OMCLs (L3 tests). In 2001, the average cost per test to perform 3000 color reactions for identification and content using 1000 TLC analyses, was US\$ 1.50 [22]. In today's market the cost of performing L2 analyses remains relatively inexpensive at about US\$2.00 per TLC test, well below the average cost of performing an L3 analysis such as content by HPLC.

9. Capability to test a wide range of therapeutic treatments: Depending on countries' needs, it is possible to develop and validate TLC methods for L2 analyses for medicines of interest. In particular, the GPHF Minilab used by countries collaborating with PQM contains the necessary methods and reference standards to perform L2 tests for more than 60 medicines from the WHO Model Lists of Essential 
Medicines [23], spanning a wide range of therapeutic classes (eg, antibiotic, antimalarial, antiretroviral, antituberculosis, analgesic, and anti-inflammatory medicines).

10. Applicability throughout the supply chain: L1 and L2 can be implemented not only at storage facilities or dispensing sites in decentralized areas, but also throughout the supply chain after registration (see below), and in ports of entry. It may be of particular benefit during procurement and distribution of medicines purchased by ministries of health to support national health programs, such as malaria, tuberculosis and HIV/SIDA.

\section{Limitations:}

1. Capability to assess only a limited number of critical quality attributes: L2 methodologies cannot asses certain critical quality attributes of solid dosage forms (e.g. dissolution, uniformity of dosage units, water content, and certain types of impurities that require a higher level of sensitivity). Failures in dissolution, in particular, may have a dramatic effect on the therapeutic value of a medicine.

2. Limited use for injectable formulations: Microbiological assessment, critical for injectables, are beyond the capabilities of L2 testing

3. Limited experience of personnel performing L2 analyses: Depending on the particular setting, high turnover of the personnel performing L2 analyses may result in personnel lacking the necessary experience to perform even these simple analytical tests. Therefore, proper steps should be implemented to enable the OMCL or other qualified institution to provide training or periodically verify some of the L2 tests results to ensure proficiency in producing data that are trustworthy and valid.

4. Inconclusive results: In certain cases, particularly for content, L2 analyses can result in inconclusive results and may require the use of more sophisticated methodology.

5. Limited support for implementing corrective actions: As mentioned above, to implement corrective actions in many countries, national regulations require testing the product with the manufacturervalidated or pharmacopeial methodology (L3) specified during registration.

\section{Integration of L1, L2, and L3 in the three-level approach}

Even as the Three-level Approach promotes L1 and L2 as worthy and effective levels of quality control in the field, L3 tests remain the ultimate arbiter of medicines quality, and the OMCL needs to be fully integrated in the process to ascertain that the results obtained through L1 and L2 testing are valid and trustworthy. In this context, the OMCL should perform two types of activities: verification testing and confirmatory testing.

Verification testing refers here to the repetition at the laboratory of the L2 screening tests performed in the field. Experienced laboratory analysts evaluate the performance of field staff by verifying the latter's results. At the laboratory, L2 testing may be conducted as it is done in the field or with more refined equipment, such as disintegration apparatus and more advanced TLC systems. In addition, performing verification tests is mandatory when the results in the field are doubtful.

Confirmatory L3 testing usually refers to analyses performed with manufacturer-validated methods or pharmacopeial methods. Within the framework of the Three-level Approach, L3 tests are performed in all samples that failed L2 and on a subset of those passing L2 screening. The reasons for that have been explained above: assessment of all quality specifications, better accuracy, and legal requirements for implementing corrective actions. In addition to those, one should add the risk of quality problems that cannot be evaluated by L2 screening tests, as explained in the following two example: a. Fixed-dose combination tablets of sulfadoxine and pyrimethamine commonly pass disintegration tests, an L2 test, but often fail pyrimethamine dissolution analysis $[12,22,24]$, included in L3 analysis for solid dosage forms. b. Artemesinin derivatives (e.g., artesunate, artemether, and others) typically pass the three quality attribute tests evaluated in L2 by TLC, but they fail to pass more sensitive tests available in $\mathrm{L} 3$ to detect low level impurities [22,24].

\section{Implementation of the three-level approach throughout the supply chain}

Marketing authorization is issued by a medicines regulatory authority approving the marketing of a medicine in a country based on safety, efficacy and quality data. For the latter, the quality specifications being established for a medicine may be confirmed by regulatory authorities through QC analysis during registration. After marketing authorization is granted, it is important to ensure that quality is maintained throughout the supply chain, and therefore it is imperative to establish processes that allow quality monitoring during procurement, distribution and at the point of use (Figure 1). Using L1 and L2 as QC tools to complement L3 analyses allows the approach to be used in assessments during procurement, distribution, and at the point of use.

The recommended level of QC analyses implemented before and after market authorization are identified accordingly (L1, L2 and L3). L1 should always be performed in all QC assessments at each of the mentioned points of implementation (registration, procurement, distribution and point of use) regardless of whether or not L2 and/or $\mathrm{L} 3$ analyses will be done.

Registration: Registration is the critical stage when medicines are approved for use and sale, and it is of utmost importance to verify that the product complies with all of the manufacturer's registration specifications. This process should be performed only by personnel at a qualified laboratory, preferentially an OMCL, using the manufacturervalidated methods or pharmacopeial methodology described in the registration dossier. Therefore, the limitations of $\mathrm{L} 2$ analyses restrict it from being used for QC during registration.

Procurement: Government agencies and other institutions that procure medicines may follow different procedures depending on the source (donations, national or foreign manufacturers, distributors, importers, international procurement organizations, nongovernmental organizations, or bilateral/multilateral aid programs). Regardless of the source of the medicine, however, good procurement practices should apply [25-27]. All procured medicines should be registered and approved for use by relevant stakeholders.

Depending on country's regulations, after a source has been selected or identified, procured medicines undergo various types of processes to assess quality. Certain countries submit all incoming lots to L3 testing, others analyze only randomly selected lots, and others perform only L1 analyses, documentary review, or a combination of these processes. Figure 2 shows application of the Three-level Approach during procurement where L2 analysis (eg, disintegration, TLC or spectroscopic methods) may be used as an alternative to L3 analysis.

Quality control via Path " $A$ " is recommended for medicines procured from manufacturers with a history of providing good 


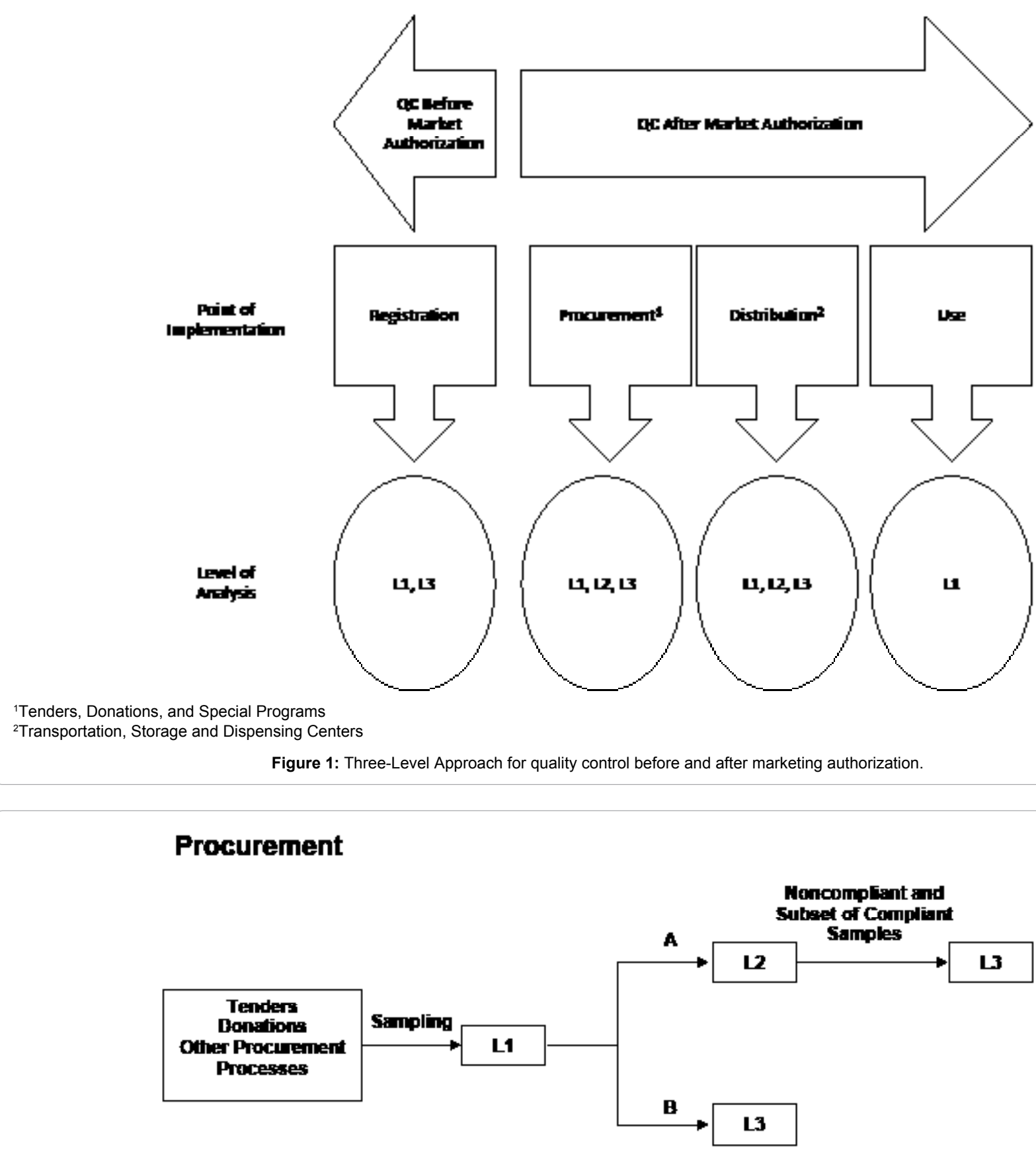

Figure 2: Three-level Approach in procurement.

quality medicines (e.g., prequalified by WHO, compliant with a Good Manufacturing Practices (GMP) audit performed by a Stringent Drug Regulatory Authority [26] or products with a history of compliant QC data). Path " $\mathrm{B}$ " may be applied when medicines procured for the first time are of unknown quality due to a lack of evidence-based QC data, or when there is evidence of quality issues due to poor GMP audit findings, previous quality failures, or intrinsic characteristics/attributes of the product exist.

Distribution: The MRA must ensure that the entire pharmaceutical market is adequately controlled and that all sectors are regulated. This includes monitoring a complex distribution chain in which medicines may circulate through multiple distributors, increasing the risks of deterioration or the introduction of counterfeits. One approach to ensure medicine quality throughout the distribution chain is to implement a medicine quality monitoring program in public health facilities and registered private pharmacies. The MRA also must police the informal or illegal sector where medicine quality issues have been found $[21,24,28,29]$. Performing L2 testing throughout various stages of the distribution chain can help safeguard against the introduction of counterfeits and aid in their identification. As a result, the risks of patients' consuming poor-quality medicines can be greatly mitigated.

Figure 3 illustrates the application of the Three-level Approach during: a) transportation and short-term storage and b) longterm storage and at dispensing centers. At various points during 


\section{Distribution}
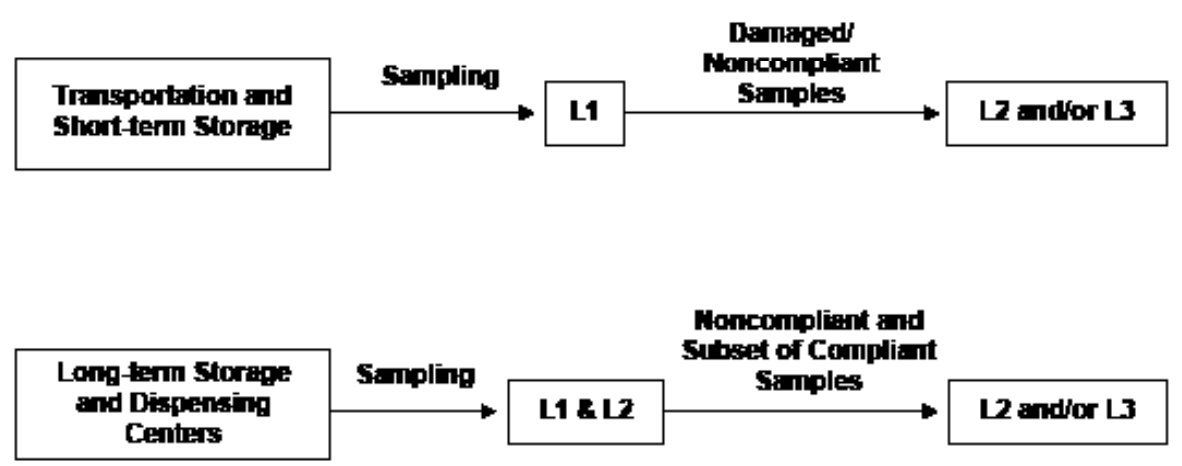

Figure 3: Three-level Approach in distribution.

transportation and short-term storage $\mathrm{L} 1$ testing should be performed to verify that the packaging and the physical characteristics of the medicines are maintained. Irregularities should be documented, and the need for further assessment via L2 and/or L3 analyses should be evaluated according to established procedures, taking into account the severity of the damage or the irregularity encountered. In longterm storage, medicines generally do not undergo QC testing before they reach their final destinations where they will be dispensed to patients. Yet these medicines may be stored for prolonged periods of time under conditions that could compromise their quality. L2 testing is a convenient tool to help determine when deteriorated medicines need to be withdrawn from circulation. Similar tests can be used at dispensing centers, particularly in remote areas where stability could be compromised because storage conditions tend to be deficient and ambient temperature or humidity are usually beyond those recommended for the medicines.

Point of use: The point of use is generally ignored during discussions of the distribution chain and quality control, yet this end point of the chain is most critical to patients. It is important to educate patients about performing L1 analysis. This simple process could identify expired medicines and those of questionable quality. The straightforward physical examination of a product can prevent medicines of potentially poor quality from being consumed. This process could provide substantial added value by making the patient part of the QA system.

\section{Implementation of the three-level approach during MQM activities}

Routine surveillance of the market is a critical activity to ensure that the medicines available to the patients, in public as well as private facilities are of good quality. The Three-level Approach is a reasonable strategy to support MQM and deliver timely results at a low-cost, particularly in remote areas and when hundreds of samples are being collected.

Figure 4 illustrates the application of visual and physical inspection (L1) and screening (L2) to detect counterfeit and substandard medicines during MQM. The figure also conceptually illustrates how the approach can reduce the number of samples submitted for L3 analysis.

Despite the need to integrate the three levels, remote locations may not have timely access to the laboratory to perform L3 analysis or may not even have the available tools to perform L2 screening tests. This should not deter relevant health authorities from implementing routine medicine quality monitoring activities using visual and physical inspection alone (L1) or in combination with screening tests (L2) to provide a minimal level of assurance. This is particularly relevant because L1 and L 2 can detect many quality issues in both substandard and counterfeit medicines. Failures in antimalarial medicines quality in the Amazon Basin were mostly detected based on L1 and L2 assessments [29].

\section{Experiences in the field utilizing the three-level approach for MQM activities}

The PQM program has successfully introduced L2 analysis as a key part of medicines quality monitoring in approximately 25 countries in Africa, South America, and Asia [21,28-32] and continues to promote adoption of the Three-level Approach in more countries around the world. Most of the more than 13,000 medicines analyzed since 2003 and included in PQM's Medicine Quality database (MQDB) [33] have been tested within the framework of this approach. Authorities in Cambodia, Laos, and Vietnam have implemented policies incorporating screening tests during routine MQM. A 2003 sampling of medicines in Cambodia found that $27 \%$ of the samples undergoing screening tests failed either TLC or disintegration [30]. Subsequently corrective actions were taken and media campaigns were developed that have led to greater public awareness, resulting in a sample failure rate of $1 \%$ in 2010 [34]. In South America, in the context of the Amazon Malaria Initiative [35], screening tests have been implemented in decentralized areas of seven countries [29]. In this study, systematic L1 assessments resulted in a better inventory of malaria medicines in governmental facilities. In addition to those countries, Guatemala and Peru have recently begun implementation of L2 as well. Based on the success of the approach, some MRAs in Central and South America are in the process of including the approach in country's MRA's regulations. The survey performed by USP Drug and Quality Information (DQI) program, the predecessor of PQM, in Senegal, Uganda and Madagascar [21] also reported on the utility of screening tests. In Senegal, the failure rates by L2 and L3 analysis were similar at $43 \%$ and $44 \%$ respectively; however, the results obtained in both Uganda and Madagascar provided evidence of the limitations of screening tests, the inability to assess dissolution and detect certain impurities. More than $52 \%$ of the failures identified in a study performed in Guyana could have been detected by L2 testing [28].

\section{Conclusions}

Testing medicines using L3 methods has traditionally been the main analytical approach for the quality control of medicines in most 
Objective: MQM at Decentralized Locations, $N=100$ Samples

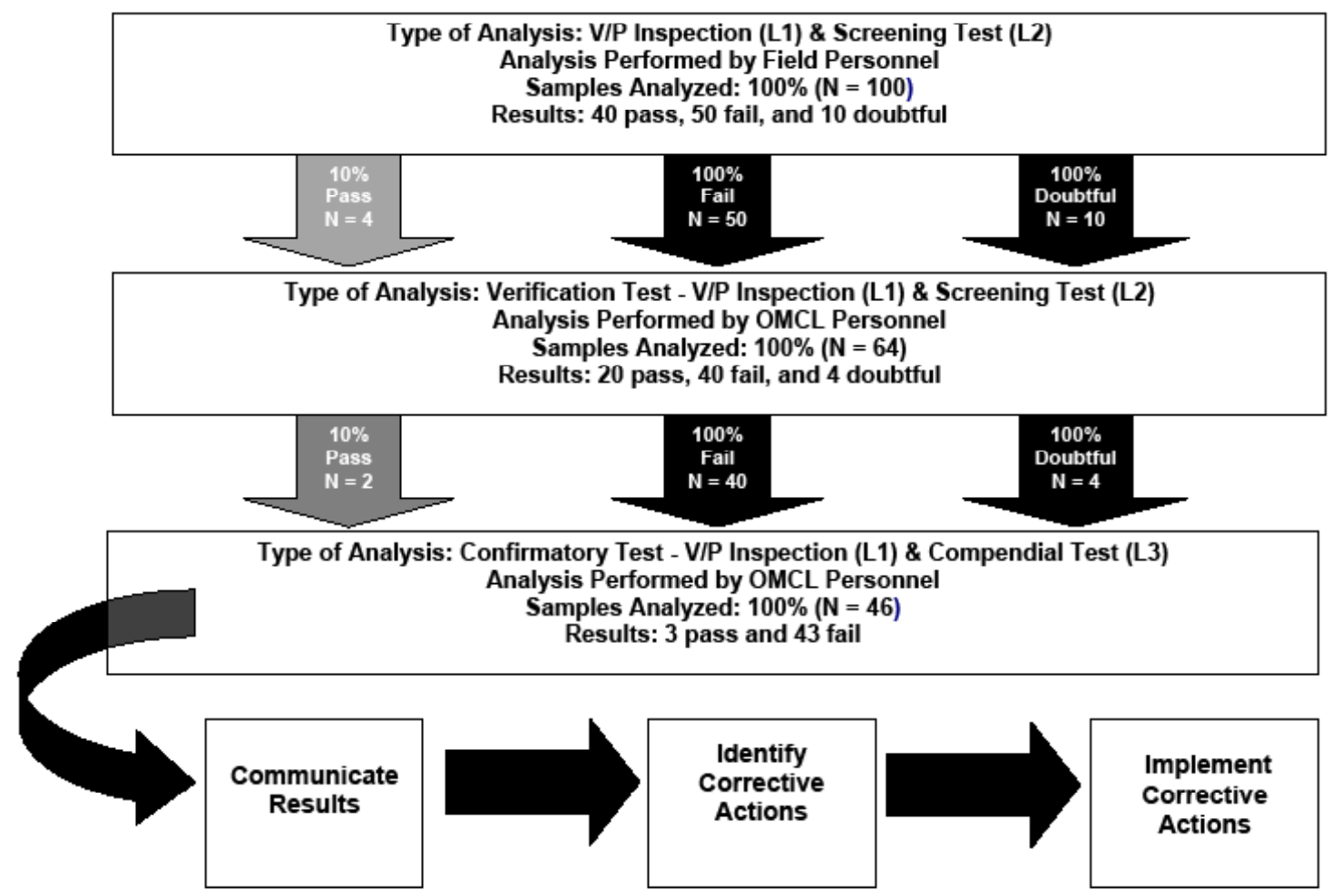

Figure 4: Application of the Three-level Approach for MQM in decentralized locations.

countries. The Three-level Approach is intended to ensure the quality of medicines in countries with limited human and financial resources by means of a staged process in which visual and physical inspection (L1) and screening (L2) analyses precede and complement manufacturervalidated and pharmacopeial methods (L3). By incorporating visual and physical inspection (L1) and screening tests (L2) as independent steps to increase analytical throughput, the Three-level Approach is capable to provide rapid QC results for a large number of samples in a cost-efficient manner. Consequently, the Three-level Approach strengthens national QA systems and allows better oversight of the pharmaceutical market.

The use of L2 screening tests needs to be complemented with L3, and should not be employed as the sole source of QC results. MRAs should be aware of these limitations, and should understand that the various quality control levels are complementary procedures that always need to be applied with proper guidelines. These guidelines help develop sampling and analysis protocols that are based on the local context and clearly define the roles and responsibilities of all the stakeholders involved.

Ultimately, implementation of the Three-level Approach will allow countries to reduce the prevalence of substandard and counterfeit medicines and will mitigate the detrimental economic and health effects associated with the consumption of poor-quality medicines.

\section{Acknowledgments}

The authors wish to thank Ms. Marilyn Foster, Mr. Stefan Schuber and Dr. Desmond Hunt for their review and editing of the article.

\section{Funding}

This work was supported by the United States Agency for International
Development (USAID) [Cooperative Agreement No. GHS-A-00-09-00003-00]. The contents are the responsibility of the Promoting the Quality of Medicines Program implemented by the United States Pharmacopeial Convention and do not necessarily reflect the views of USAID or the United States Government.

\section{References}

1. WHO (2012) Medicines: spurious/falsely-labelled/falsified/counterfeit (SFFC) medicines: Fact sheet Number 275

2. Newton PN, Green MD, Fernández FM, Day NP, White NJ (2006) Counterfeit anti-infective drugs. Lancet Infect Dis 6: 602-613.

3. Caudron JM, Ford N, Henkens M, Macé C, Kiddle-Monroe R, et al. (2008) Substandard medicines in resource-poor settings: a problem that can no longer be ignored. Trop Med Int Health 13: 1062-1072.

4. Petralanda I (1995) Quality of antimalarial drugs and resistance to Plasmodium vivax in Amazonian region. Lancet 345: 1433.

5. Newton PN, McGready R, Fernandez F, Green MD, Sunjio M, et al. (2006) Manslaughter by fake artesunate in Asia--will Africa be next? PLoS Med 3: e197.

6. Institute of Medicine (2013) Countering the problem of falsified and substandard drugs.

7. Tadeg H, Berhane $Y$ (2012) Substandard and counterfeit antimicrobials recent trends and implications to key public health interventions in developing countries. East Afr J Public Health 9: 85-89.

8. Kohler JC, Pavignani E, Michael M, Ovtcharenko N, Murru M, et al. (2012) An examination of pharmaceutical systems in severely disrupted countries. BMC Int Health Hum Rights 12: 34

9. Alfadl AA, Abdoon S, Elamin M, Elnabi NG (2006) Quality of antimalarial drugs in Sudan: Results of post-marketing surveillance. Sudanese J Public Health 1: $108-11$.

10. Nayyar GM, Breman JG, Newton PN, Herrington J (2012) Poor-quality antimalarial drugs in southeast Asia and sub-Saharan Africa. Lancet Infect Dis 12: 488-496. 
Citation: Pribluda VS, Barojas A, Coignez V, Bradby S, Dijiba Y, et al. (2014) The Three-Level Approach: A Framework for Ensuring Medicines Quality in Limited-Resource Countries. Pharmaceut Reg Affairs 3: 117. doi:10.4172/2167-7689.1000117

11. Newton PN, Green MD, Mildenhall DC, Plançon A, Nettey H, et al. (2011) Poor quality vital anti-malarials in Africa - an urgent neglected public health priority. Malar J 10: 352.

12. Amin AA, Kokwaro GO (2007) Antimalarial drug quality in Africa. J Clin Pharm Ther 32: 429-440.

13. O'Connell KA, Gatakaa H, Poyer S, Njogu J, Evance I, et al. (2011) Got ACTs? Availability, price, market share and provider knowledge of anti-malarial medicines in public and private sector outlets in six malaria-endemic countries. Malar J 10: 326.

14. Seear M (2012) Pharmaceutical quality: an urgent and unresolved issue. Lancet Infect Dis 12: 428-429.

15. WHO (2010) Assessment of medicines regulatory systems in sub-Saharan African countries. Accessed 20 February 2014

16. Reddy D, Banerji J (2012) Counterfeit antimalarial drugs. Lancet Infect Dis 12 829

17. Kaplan WA, Laing $R$ (2003) Paying for pharmaceutical registration in developing countries. Health Policy Plan 18: 237-248.

18. USP (2013) PQM: Promoting the Quality of Medicines program

19. Thin Layer Chromatographic Tests. GHFP-Minilab Manual.

20. Hajjou M, Qin Y, Bradby S, Bempong D, Lukulay P (2013) Assessment of the performance of a handheld Raman device for potential use as a screening tool in evaluating medicines quality. J Pharm Biomed Anal 74: 47-55.

21. USP DQI (2010) United States Pharmacopeia Drug Quality and Information Program. 2010. Survey of the quality of selected antimalarial medicines circulating in Madagascar, Senegal, and Uganda.

22. Jahnke RWO, Kusters G, Fleischer K (2001) Low-cost quality assurance of medicines using the GPHF-Minilab. Drug Inf J 35: 941-945.

23. WHO (2011) WHO Model List of Essential Medicines 2011. Geneva: World Health Organization. Accessed 20 February 2014.

24. WHO (2011) Survey of the quality of selected antimalarial medicines circulating in six countries of sub-Saharan Africa. Accessed 20 February 2014.
25. WHO (1999) Operational Principles for Good Pharmaceutical Procurement Accessed 20 February 2014.

26. World Bank. 2005. Strengthening procurement capacities in developing countries. Accessed 20 February 2014.

27. WHO 2011. Guideline on Submission of Documentation for Prequalification of Multisource (generic) Finished Pharmaceutical Products (FPPs) approved by Stringent Regulatory Authorities(SRAs) in the regions of the International Conference on Harmonization of Technical Requirements for Registration of Pharmaceuticals for Human Use (ICH). Accessed 20 February 2014.

28. Evans L 3rd, Coignez V, Barojas A, Bempong D, Bradby S, et al. (2012) Quality of anti-malarials collected in the private and informal sectors in Guyana and Suriname. Malar J 11: 203

29. Pribluda VS, Barojas A, Añez A, López CG, Figueroa R, et al. (2012) Implementation of basic quality control tests for malaria medicines in Amazon Basin countries: results for the 2005-2010 period. Malar J 11: 202.

30. Lon CT, Tsuyuoka R, Phanouvong S, Nivanna N, Socheat D, et al. (2006) Counterfeit and substandard antimalarial drugs in Cambodia. Trans R Soc Trop Med Hyg 100: 1019-1024.

31. Phanouvong S, Raymond C, Krech L, Dijiba Y, Mam B, et al. (2013) The quality of antimalarial medicines in western Cambodia: a case study along the ThaiCambodian border. Southeast Asian J Trop Med Public Health 44: 349-362.

32. Phanouvong S, Dijiba Y, Vijaykadga S, Raymond C, Krech L, et al. (2013) The quality of antimalarial medicines in eastern Thailand: a case study along the Thai-Cambodian border. Southeast Asian J Trop Med Public Health 44: 363-373.

33. Krech L, El-Hadri L, Evans L; Fouche T, Hajjou M, et al. (2014) MQDB: A Public and Freely Accessible Online Medicines Quality Database. Bull World Health Organ 2014;92:2-2A.

34. Lukulay PH, Coignez V, Pribluda V, Krech L (2011) Three-Level Approach: A Risk-Based, Cost-Effective Approach to Medicines Quality Monitoring in Lowand Middle-Resource Countries [Internet].

35. AMI (2013) Amazon Malaria Initiative: AMI. 